\title{
Gut-associated Lymphoid Tissue (GALT) Carcinoma in Ulcerative Colitis
}

\author{
CARLOS A. RUBIO ${ }^{1}$, GIOVANNI DE PETRIS ${ }^{2}$ and GIACOMO PUPPA ${ }^{3}$ \\ ${ }^{1}$ Department of Pathology, Karolinska Institute and University Hospital, Stockholm, Sweden; \\ ${ }^{2}$ Department of Gastrointestinal Pathology, Arizona Gastroenterology Associates, Scottsdale, AZ, U.S.A.; \\ ${ }^{3}$ Division of Pathology, Geneva University Hospital, Geneva, Switzerland
}

\begin{abstract}
Background: In ulcerative colitis (UC), the majority of colorectal carcinomas (CRC) arise in the vast colorectal mucosal domain built with mucus-producing goblet cells and columnar cells. Conversely, CRC in UC rarely evolve in the tiny, spotty gut-associated lymphoid tissue (GALT) mucosal domain. Here we review the four reported cases of colonic carcinoma developing in GALT mucosa in UC, searching for possible precursor lesions connected with the evolution of these tumours. Materials and Methods: The clinical history, age, gender, endoscopic descriptions, and the pathology (localization, gross and histological descriptions of the luminal surface) of the four $U C-G A L T$ carcinomas reported in the literature were reviewed. Results: The luminal surface in three out of the four carcinomas revealed conventional (tubular/villous) adenomas or high-grade dysplasia. All four UC-GALTcarcinomas were detected at an early stage (TINO). Conclusion: GALT carcinomas do occur, albeit infrequently, in patients with UC. The finding that three out of the four GALT carcinomas on record were covered by conventional adenomas or by high-grade dysplasia strongly suggests that non-invasive conventional neoplasias might often precede GALT carcinomas in UC.
\end{abstract}

The currently accepted concensus is that sporadic colorectal carcinomas (CRC) develop via the conventional (tubular or villous) adenoma-carcinoma pathway (1) or the serrated

This article is freely accessible online.

Correspondence to: Carlos A. Rubio, MD, Ph.D., Gastrointestinal and Liver Pathology Research Laboratory, Department of Pathology, Karolinska Institute and University Hospital, 17176, Stockholm, Sweden. Tel: +46 851774527, Fax: +46 851774524, e-mail: Carlos.Rubio@ki.se

Key Words: Gut-associated lymphoid tissue, GALT, colorectal mucosa, GALT carcinoma, ulcerative colitis. adenoma-carcinoma pathway (2). Both these trails of 919progression evolve in the vast colorectal mucosal domain built with mucus-producing goblet cells and columnar cells. Sporadic CRC evolving in the remnant, tiny and spotty, gutassociated lymphoid tissue (GALT) mucosa are rare, a trail recently referred to as the third pathway of colorectal carcinogenesis (3).

Patients with UC are at increased risk of developing CRC, particularly those with early onset or with total-longstanding colitis (4). In contrast to sporadic CRC, CRC in UC might be preceded by different epithelial lesions: i) dysplasia in flat mucosa (5), ii) UC-related conventional (tubular or villous) adenomatous growth (6), iii) UC-related serrated adenomatous growth $(7,8)$, iv) dysplasia in UC-related subtle villous changes (9), v) conventional (tubular or villous) adenomas on top of GALT (3), or vi) UC-unrelated, synchronously growing, age-dependent, sporadic conventional or serrated adenomas (8). It should be understood that these precursor lesions also develop in the vast colorectal mucosa built with mucusproducing goblet cells and columnar cells. When no precursor lesions are found in early UC carcinomas, the term de novo carcinoma has been used $(8,10)$. In similarity to the sporadic counterpart, CRC in UC rarely evolves in GALT mucosal domains. In a lecture 114 years ago, at gross examination, Ball suspected a carcinoma arising in an organized lymphoid follicle: These were his words: "We occasionally meet with small tumours which clinically resemble closely simple adenomata, but which upon microscopic examination prove to be composed of lymphoid tissue instead of the epithelial elements" (11).

In 1954, Cuthbert Dukes described a histological lesion in the submucosa characterized by "misplaced" colonic epithelium in patients with UC (12). Dukes submitted that the misplaced epithelium was the result of mucosal repair following regeneration of a mucosal ulcer, and that the epithelium detached and buried in the submucosal would encourage cancer development (12). Following the suggestion of Dukes, the frequency of misplaced (i.e. ectopic) colonic mucosa in 62 colectomy specimens was 

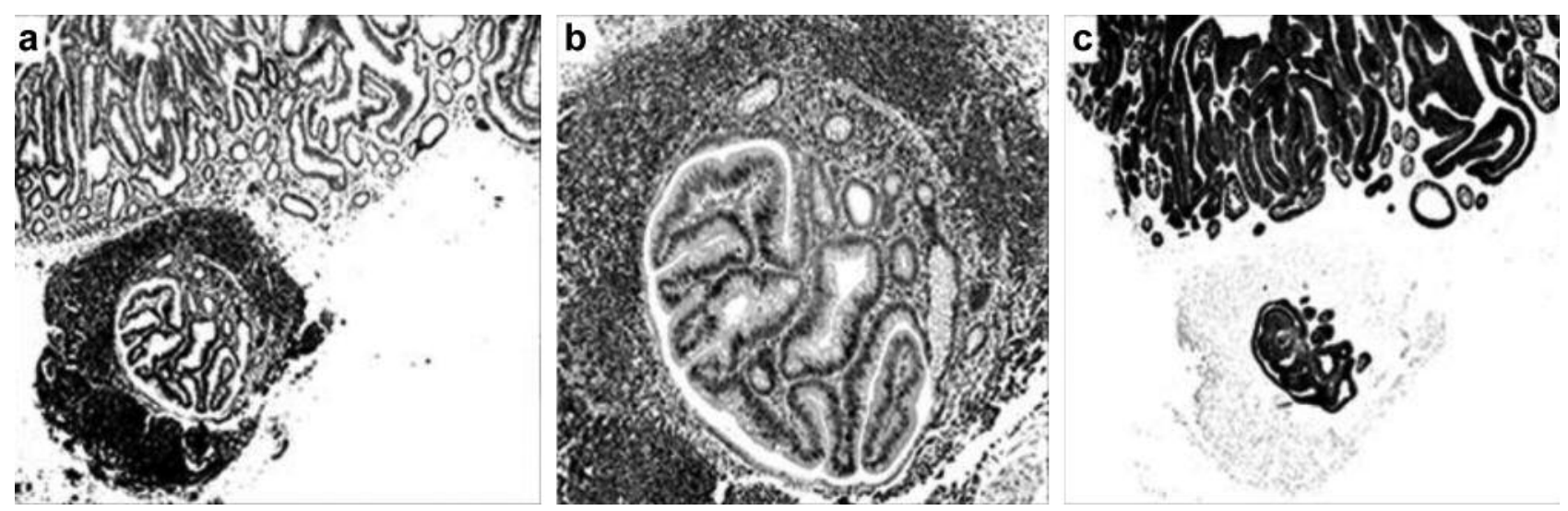

Figure 1. a: Villous adenoma on top of a gut-associated lymphoid tissue (GALT) carcinoma (H\&E magnification $\times 4)$. b: Detail from GALT carcinoma (H\&E magnification $\times 10)$. c: GALT carcinoma immunostained with the anti-cytokeratin antibody MNF 116 to highlight the epithelial structures of the villous adenoma on top and the GALT carcinoma in the submucosa (MNF 116 magnification $\times 4$ ).

Table I. Clinical data and descriptive characteristics in four carcinomas arising in gut-associated lymphoid tissue in four patients with ulcerative colitis reported in the literature.

\begin{tabular}{lccccc}
\hline Year (ref.) & Senior author & Localization & Gross description of the surface & Histology at the surface & Age, years/gender \\
\hline $1984(13)$ & Rubio & Left colon & Irregular plaque-like lesion & Chronic inflammation & 57/M \\
$2002(14)$ & Rubio & Right colon & Sessile polyp & Adenoma HGD & $53 /$ F \\
$2008(15)$ & Stewart & Right colon & Polypoid lesion & HGD & $70 /$ M \\
$2013(16)$ & Rubio & Transverse colon & Polypoid lesion & Adenoma HGD & $68 /$ F \\
\hline
\end{tabular}

HGD: High-grade dysplasia.

reported in 1984 (13). One or more foci of misplaced mucosa was found in $72 \%$ of the 22 colectomies from patients with UC, in $55 \%$ of 20 from patients with Crohn's disease-associated colitis, and in none of 20 from patients without inflammatory bowel disease. In one patient with UC, an adenocarcinoma surrounded by nodular lymphoid tissue invading the submucosa was found. This histological characteristic fulfilled the criteria of colonic GALT carcinoma (13). Since then, three additional cases of GALT carcinomas evolving in UC have been reported (14-16).

The purpose of this communication was to review the four cases of GALT carcinoma in patients with UC in the literature, searching for possible precursor lesions connected with the evolution of this carcinoma in UC.

\section{Materials and Methods}

Literature review. The search was performed using the PubMed electronic database.

The narratives of the clinical history (age, gender, endoscopic descriptions), and the pathology (localization, gross and histological descriptions of the luminal surface) of the four cases of colorectal GALT carcinomas in UC on record, were reviewed.

\section{Results}

The results are presented in Table I.

Clinical data. The mean age of the four patients was 62 years (range $=53-70$ years). Two out of the four cases were males and the remaining two, females. All four cases had protracted UC.

Pathological description. Localization. In two out of the four cases, the GALT carcinoma was located in the right colon, one in the transverse colon and the remaining one in the left colon.

Gross description of the surface. Out of the four GALT carcinomas, three were reported at endoscopic examination as being polyp/polypoid lesions and the remaining one as an irregular plaque-like lesion. 
Histology of the luminal surface. Histology of the luminal surface covering the GALT carcinoma in two out of the four cases revealed conventional adenomas (Figure 1), one disclosed high-grade dysplasia, and the remaining one mucosa with chronic inflammation. The four GALT carcinomas were confined to the submucosa. Neither of the two adenomas described in Table I showed poor glandular differentiation, budding or lymphovascular invasion.

\section{Discussion}

In patients with UC, the vast majority of CRCs evolve in the GALT-free mucosa. In contrast, CRC arising in GALT domains in patients with UC occur much less frequently (17).

The first colonic GALT carcinoma in a patient with UC was reported in 1984 (13). Since then, three additional cases of GALT carcinoma in patients with UC have been published (1416). Notably, three out of the four UC-GALT carcinomas were covered by conventional adenomas or high-grade dysplasia, strongly suggesting that non-invasive conventional neoplasias might precede GALT carcinoma in UC. All four UC-GALT carcinomas were detected at an early phase (T1N0).

The low frequency of UC-GALT-carcinomas could be due to the fact that only minute areas of the colorectal mucosa are occupied by GALT domains (17).

As a corollary, GALT carcinomas do occur, albeit infrequently, in patients with UC. Studies of the molecular pathway in GALT carcinomas in UC are limited by the rarity of these tumours. As a light at the end of the tunnel, it should be mentioned that GALT carcinomas were found in $53 \%$ of colonic GALT mucosal domains in Sprague-Dawley rats treated with the colonotropic carcinogen 1,2 dimethylhydrazine (18). This animal model would permit further investigation into the molecular signals required for the development of GALT carcinomas in rats having synchronously experimentally induced ulcerative colitis $(19,20)$.

\section{Conflicts of Interest}

The Authors have no conflict of interest to declare in regard to this study.

\section{References}

1 Jackman RJ and Mayo CW: The adenoma-carcinoma sequence in cancer of the colon. Surg Gynecol Obstet 93: 327-330, 1951.

2 Jass JR: Serrated adenoma and colorectal cancer. J Pathol 187: 499-502, 1999.

3 Rubio CA, Puppa G, de Petris G, Kis L and Schmidt PT: The third pathway of colorectal carcinogenesis. J Clin Pathol, 2017. doi 10.1136/jclinpath-2017-204660. [Epub ahead of print].

4 Odze RD: Pathology of dysplasia and cancer in inflammatory bowel disease. Gastroenterol Clin North Am 35: 533-552, 2006.
5 Lennard-Jones JE, Melville DM, Morson BC, Ritchie JK and Williams CB: Precancer and cancer in extensive ulcerative colitis: findings among 401 patients over 22 years. Gut 31: 800806, 1990.

6 Odze RD: Adenomas and adenoma-like DALMs in chronic ulcerative colitis: a clinical, pathological, and molecular review. Am J Gastroenterol 94: 1746-1750, 1999.

7 Rubio CA, Befrits R, Jaramillo E, Nesi G and Amorosi A: Villous and serrated adenomatous growth bordering carcinomas in inflammatory bowel disease. Anticancer Res 20: 4761-4764, 2000.

8 Rubio CA: Serrated neoplasias and de novo carcinomas in ulcerative colitis: a histological study in colectomy specimens. J Gastroenterol Hepatol 22: 1024-1031, 2007.

9 Hamamoto N, Rubio CA, Befrits R, Toyoda H and Jaramillo E: Magnifying endoscopy in upper and lower GI tract: Subtlle villous changes detected at endoscopy in patients with inflammatory bowel disease. Digestive Endosc 17: S34-S39, 2005.

10 Hornick JL, Farraye FA and Odze RD: Clinicopathologic and immunohistochemical study of small apparently "de novo" colorectal adenocarcinomas. Am J Surg Pathol 31: 207-215, 2007.

11 Ball CB: The Erasmus Wilson Lectures on adenoma and adenocarcinoma of the rectum. Br Med J 1: 413-416, 1903.

12 Dukes CE: The surgical pathology of ulcerative colitis. Ann R Coll Surg Engl 14: 389-400, 1954.

13 Rubio CA: Ectopic colonic mucosa in ulcerative colitis and in Crohn's disease of the colon. Dis Colon Rectum 27: 182-186, 1984.

14 Rubio CA and Talbot I: Lymphoid-associated neoplasia in herniated colonic mucosa. Histopathology 40: 577-579, 2002

15 Stewart C, Hillery S and Newman N: Dome-type carcinoma of the colon. Histopathology 53: 231-233, 2008.

16 Rubio CA, Befrits $\mathrm{R}$ and Ericsson $\mathrm{J}$ : Carcinoma in gutassociated lymphoid tissue in ulcerative colitis: Case report and review of literature. World J Gastrointest Endosc 5: 293-296, 2013.

17 Rubio CA: Colorectal carcinogenesis from gut-associated lymphoid tissue. Clinical and experimental documentation. Br J Med Med Res 21: 1-12, 2017.

18 Rubio CA: The histogenesis of the third pathway of colonic carcinogenesis in rats. Anticancer Res 37: 1039-1042, 2017.

19 Yang Y, Zhu X, Qin Y, Chen G, Zhou J, Li L, Guan J, Ma L and Xue Y, Li C: The Anti-inflammatory effect of guchangzhixie-pill by reducing colonic EC cell hyperplasia and serotonin availability in an ulcerative colitis rat model. Evid Based Complement Alternat Med 2017: 8547257, 2017.

20 Suluvoy JK, Sakthivel KM, Guruvayoorappan C and Berlin Grace VM: Protective effect of Averrhoa bilimbi L. fruit extract on ulcerative colitis in Wistar rats via regulation of inflammatory mediators and cytokines. Biomed Pharmacother 91: 1113-1121, 2017. 